\title{
Confinement d'un plasma par un systeme polyedrique à courant alternatif
}

\author{
R. KELLER et I. R. Jones \\ Laboratoire de Recherches sur la Physique des Plasma, Fonds National Suisse de la Recherche \\ Scientifique, Lausanne, Suisse
}

(Z. Naturforschg. 21 a, 1085-1089 [1966] ; reçu le 2 mars 1966)

Dedié au Professeur Dr. W. Gentner à l'occasion de son soixantième anniversaire

\begin{abstract}
Un plasme confiné par une configuration polyédrique est exploré au moyen d'une sonde piézoélectrique et photographié avec une caméra électronique. La configuration de champ est formée de deux systèmes de conducteurs, l'un octaédrique et l'autre cubique tronqué, alimentés par des courants de haute fréquence déphasés de $90^{\circ}$ l'un par rapport à l'autre. Le tout se comporte en moyenne comme un cusp possédant un grand nombre de points de fuite répartis sur une sphère. On observe une onde sphérique convergente. Le rendement du transfert d'énergie au plasma est élevé.
\end{abstract}

Le confinement magnétique d'un plasma nécessite la création d'une configuration de champ qui présente le moins possible de régions de fuites. En outre, le champ doit posséder une propriété stabilisatrice envers les déformations hydromagnétiques de la surface. Par exemple, il y a stabilité lorsque le champ magnétique (sa valeur efficace s'il est alternatif) augmente le long d'un chemin s'éloignant vers l'extérieur de la couche limite. La géométrie en forme de cusp est un exemple typique de configuration stable.

Beaucoup d'études ont été entreprises ${ }^{1,2,3}$ en vue d'obtenir des configurations satisfaisant au critère de confinement (p. ex. miroirs magnétique et géométries toroïdales) ou au critère de stabilisation (p. ex. géométries dites «cuspidées», en forme de puit magnétique, et stabilisation au moyen de conducteurs multipolaires de Ioffe).

Le présent travail concerne l'étude de certaines propriétés d'un plasma créé dans une configuration caractérisée par un grand nombre de cusps ponctuels répartis régulièrement autour d'un volume quasisphérique. La bouteille magnétique est composée de deux systèmes de conducteurs polyédriques sans couplage mutuel, l'un octaédrique et l'autre cubique tronqué, alimentés par des courants de haute fréquence déphasés de $90^{\circ}$ l'un par rapport à l'autre. Les deux bobinages sont orthogonaux dans l'espace et dans le temps. La configuration est à la fois stable par rapport aux déformations hydromagnétiques et plus étanche qu'un cusp bicônique.

1 T. K. Allen et I. J. Spalding, Phys. Fluids 8, 2032 [1965].

2 S. N. Breus, V. N. Kourdumov, M. L. Lévine, C. M. Osovetz, N. Y. Popov, K. V. Khodataïev et V. P. Chimtchouk, Etude dans „Delta-2“ de l'interaction d'une onde progressive électro-magnétique de haute fréquence et d'un plasma.

\section{Propriété des systèmes polyèdriques}

1. Le système octaédrique. Le arêtes de l'octaèdre (voir Figure $1 \mathrm{a}$ ) sont parcourues par un courant dont le sens est indiqué par des flèches. L'octaédre possède deux types d'axes de symétrie.

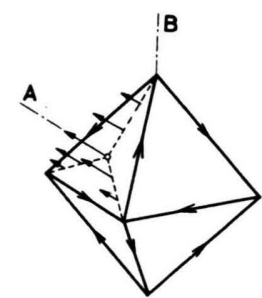

Fig. 1 a. Système octaèdrique.

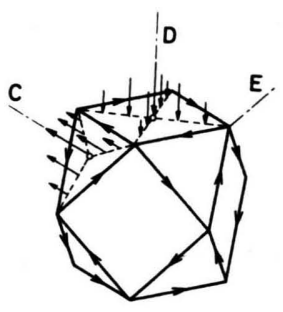

Fig. 1 b. Système cubique tronqué.
Les axes $A$, perpendiculaires aux faces, contiennent un cusp ponctuel. Les axes $B$ passant par les sommets sont des lignes à champ nul. Les cusps ponctuels se prolongent par des cusps linéaires qui décroissent progressivement jusqu'àux axes $B$. Dans la Fig. 1 a les lignes de champ sont schématisées par des flèches.

2. Le système cubique tronqué. Ce polyèdre possède trois types d'axes de symétrie (voir Fig. 1 b) . Les axes $C$ et $D$ sont perpendiculaires aux faces triangulaires et carrées. Ils contiennent un cusp ponctuel. De chacun des cusps ponctuels s'étend un cusp linéaire allant vers les axes $E$ qui sont des lignes à champ nul.

Les deux polyèdres imbriqués l'un dans l'autre forment une configuration qui, lorsqu'elle est ali-

Conférence sur la Physique des plasmas et la Recherche concernant la Fusion nucléaire contrôlée. Mémoire CN-21 /219. Culham, 6-10 septembre 1965.

${ }^{3}$ M. S. Ioffe et R. I. Sobolev, J. Nucl. Energy C, 18, 501 [1965]. 
mentée en courant biphasé, passe deux fois par période de l'état octaédrique à l'état cubique et vice versa. Les axes à champ nul sont donc couverts par des cusps ponctuels alternatifs, tandis que les cusps linéaires changent de place rapidement. La configuration ainsi produite possède 14 cusps répartis uniformément sur la surface d'une sphère. Le va-et-vient rapide d'un cusp linéaire diminue les fuites du plasma. Si la fréquence est suffisamment élevée les fuites sont probablement éliminées complètement. L'idée de faire osciller la ligne de fuite d'un cusp au moyen d'un champ alternatif a été mentionnée sous référence ${ }^{4}$.

Faisons une comparaison très qualitative des pertes d'un cusp bicônique avec notre bouteille magnétique. Lors d'un confinement haute fréquence le champ magnétique pénètre dans le plasma jusqu'à une profondeur limitée. Le déclin du champ est généralement exponentiel et à une chute d'un facteur $e$ correspond une profondeur qu'on appelle épaisseur de peau. Dans le cas idéal d'une couche limite infiniment mince, la surface du plasma se termine par une pointe aigüe située sur l'axe du cusp ponctuel. Il est raisonnable d'admettre que le rétrécissement maximum dans le cas d'une couche limite réelle est de l'ordre de grandeur de l'épaisseur de peau. Ainsi, le plasma s'échappe à travers un trou de rayon égal à cette grandeur. De façon analogue le plasma s'échappe à travers une fente de largeur égale à 2 fois l'épaisseur de peau le long d'un cusp linéaire. L'aire totale des ouvertures d'un cusp bicônique est donnée par $2 \pi \delta^{2}+2 \pi R \cdot 2 \delta$ où $\delta$ est l'épaisseur de peau et $R$ le rayon de l'équateur. Pour notre bouteille magnétique l'aire totale est de $14 \pi \delta^{2}$. Les pertes sont égales lorsque $R=3 \delta$. A volume égal, notre bouteille magnétique possède de meilleures propriétés de confinement dès que $R$ est supérieur à $3 \delta$. Dans l'expérience décrite ici, l'épaisseur de peau est de l'ordre du millimètre.

\section{Le dispositif expérimental}

La réalisation du bobinage polyédrique disposé autour d'un ballon de verre est représentée par la Figure 2. Afin de diminuer l'inductivité les conducteurs sont composés de deux parallèles. Les triangles du système cubique tronqué sont agrandis au détriment

${ }^{4}$ S. Glasstone and R. H. Lovberg, Controlled Thermonuclear Reactions. D. van Nostrand Co. Inc., Princeton 1960, paragraphe 11.30 .

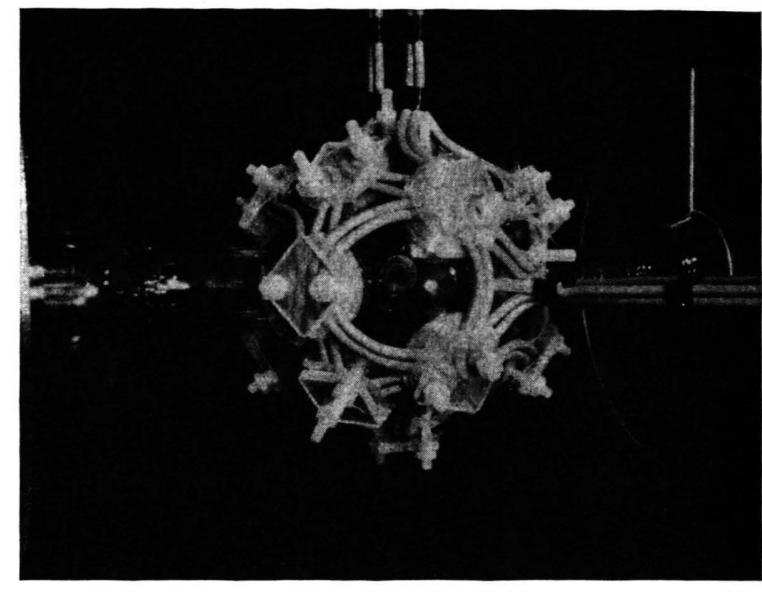

Fig. 2. Vue du bobinage polyédrique.

des quadrilatères de façon à ce que la surface totale des uns et des autres soit égale. Ainsi un même champ magnétique existe la région centrale des triangles et des quadrilatères, puisque le flux entrant par les uns est nécessairement égal au flux sortant par les autres. Le rayon intérieur du ballon de verre est de $50 \mathrm{~mm}$ et les conducteurs sont disposés sur un rayon moyen de $60 \mathrm{~mm}$.

La Figure 3 montre le schéma électrique. L'octaèdre et le cube sont alimentés par deux générateurs à lignes synchronisés (voir réf. ${ }^{5}$ ). Le cube est déphasé de $90^{\circ}$

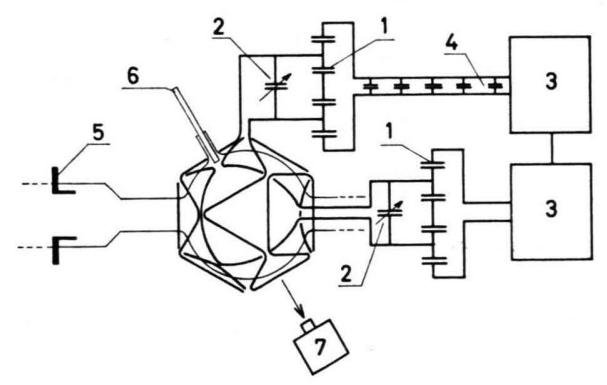

Fig. 3. Schéma électrique. 1 Condensateurs de 1600 pF, 2 Condensateurs variables 0 à 450 pF, 3 Générateurs HF synchronisés, 4 Ligne à retard quart d'onde, 5 Electrode de préionisation, 6 Sonde de pression, 7 Caméra électronique.

au moyen d'une ligne quart d'onde. L'accord des circuits est obtenu par des condensateurs variables sous vide et le contrôle du déphasage par la méthode de Lissajous. Voici les valeurs électriques dans l'ordre: système cubique puis octaédrique:

Courant efficace maximum $1680 \mathrm{~A} ; 1930 \mathrm{~A}$.

Tension efficace maximum $17,9 \mathrm{kV} ; 17,2 \mathrm{kV}$.

Capacité totale à l'accord $1600 \mathrm{pF} ; 1910 \mathrm{pF}$.

5 R. Keller, Helv. Phys. Acta 38, 328 [1965]. 
Le générateur est modulé en amplitude, sa puissance augmente quasi linéairement avec le temps pour atteindre $13 \mathrm{MW}$ à la $10 \mathrm{e}$ période. La raison pour laquelle il est judicieux de produire des trains d'ondes à amplitude croissante est expliquée sous référence ${ }^{6}$ qu'on peut résumer ainsi en quelques mots: un champ magnétique alternatif chauffe le plasma violemment. Pour que le chauffage ait lieu en équilibre de pression, cela implique qu'au fur et à mesure que la température augmente, le champ doit augmenter de telle sorte que la pression magnétique contrebalance constamment la pression du gaz.

Un banc de préionisation délivre un courant amorti de façon critique. La durée de l'impulsion est de $10 \mu$ sec et les générateurs HF sont enclenchés $20 \mu \mathrm{sec}$ après la cessation du courant de préionisation.

\section{Mesure de la pression}

Un embout de verre soudé au récipient sphérique et orienté selon un axe principal du polyèdre permet la fixation d'une sonde de pression. Le principe de cette sonde a été discuté dans réf. ${ }^{7}$ et une description détaillée d'une sonde similaire à celle qui est utilisée dans ce travail a été donnée dans réf. ${ }^{8}$. La barre de céramique qui constitue le guide d'onde acoustique est formée de deux pièces de $3 \mathrm{~mm}$ de diamètre, la partie dont l'extrémité penètre dans le plasma a une longueur de $15 \mathrm{~cm}$ et l'autre une longueur de $9 \mathrm{~cm}$. La pastille piézoélectrique est collée entre les deux pièces et le tout est maintenu librement dans une tube de verre. Les extrémités du tube et de la barre sont à fleur et il n'y a pas de joint d'étanchéité à cet endroit. Pour obtenir l'isolation entre le plasma et l'élément piézoélectrique on évacue le tube de verre par son extrémité opposée. Le débit d'hydrogène qui s'écoule entre la barre et le tube est insignifiant. L'appareil a été étalonné dans un tube de choc. Citons comme exemple un travail analogue effectué dans un $Z$-pinch, publié dans ce même journal ${ }^{9}$.

Le résultat des mesures est reporté dans les Figures 4, 5 et 6 . L'abscisse indique la longueur de pénétration de la sonde dans le ballon de verre. Chaque courbe représente la pression à un instant donné, en unités MKS. L'évolution des courbes révèle une onde sphérique convergente. La dissymétrie des courbes indique clairement un mouve-

6 R. Keller, Mesure du Vecteur de Propagation à l'Intérieur d'un Plasma Confiné et Chauffé par un Champ de Haute Fréquence. Rapport LRP 15/65, Lausanne.

7 I. R. Jones, A Review of the Pressure Bar Technique for Measuring Transient Pressures. Report No TDR-594 (1208-01) TR-3. Aerospace Corporation, El Segundo, Calif. (June 1961).

8 I. R. Jones, Pressure Bar and Photographic Measurements on a High Frequency Theta-Discharge. Rapport LRP 21/66, Lausanne.

9 K. Büchl, Z. Naturforschg. 19 a, 690 [1964].

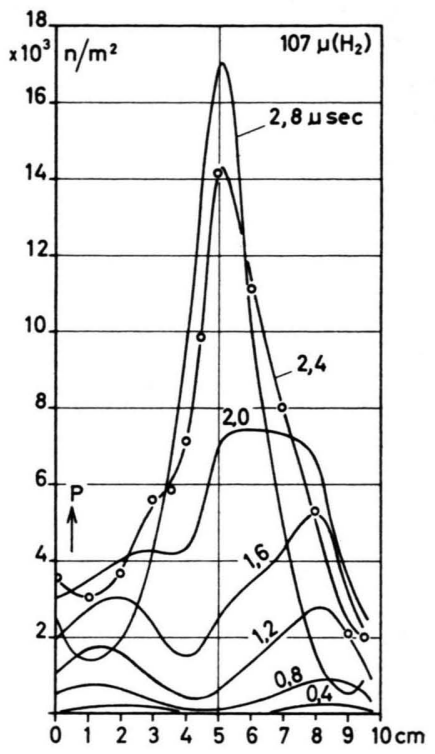

Fig. 4. Mesure de la pression. En abscisse: pénétration de la sonde.

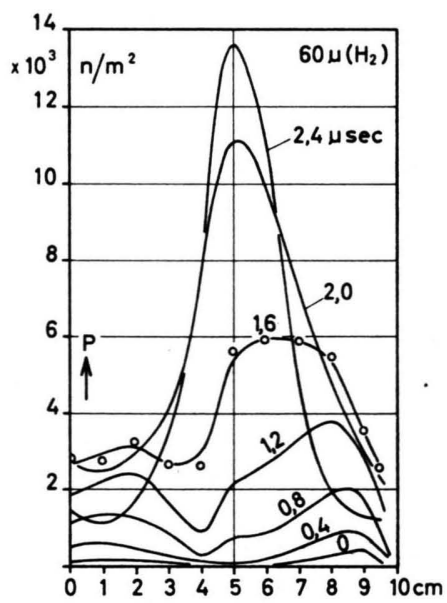

Fig. 5. Mesure de la pression. En abscisse: pénétration de la sonde.

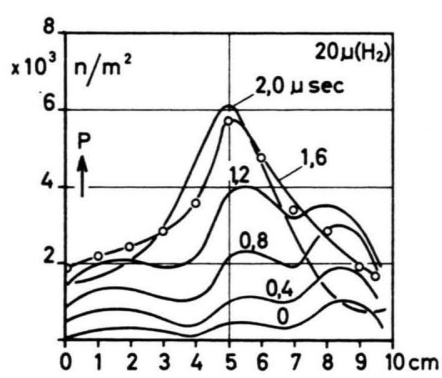

Fig. 6. Mesure de la pression. En abscisse: pénétration de la sonde. 
ment radial de masse. Une interprétation approfondie des mesures n'est pas facile. Lorsque la barre dépasse le centre, elle mesure la somme de la pression cinétique du gaz et de la pression dynamique causée par le mouvement radial de masse. Inversément, lorsque la sonde ne parvient pas jusqu'au centre, elle mesure une pression qui est quelque peu inférieure à la pression cinétique du gaz. Si le nombre de $\mathrm{M}_{\mathrm{ACH}}$ est relativement petit, on ne fera pas une grande erreur en posant la pression cinétique $p$ du plasma égale à la moyenne des pressions mesurées à égale distance en deçà et au-delà du centre. En intégrant cette pression moyenne sur le volume on obtient l'énergie

$$
E \cong \frac{3}{2} \int_{0}^{\mathrm{R}} p \cdot 4 \pi r^{2} \mathrm{~d} r
$$

où $R$ est le rayon de la sphère. Cette énergie est reportée en fonction du temps dans la Figure 7 pour les 3 pressions de remplissage $107 \mu, 60 \mu$ et $20 \mu$.

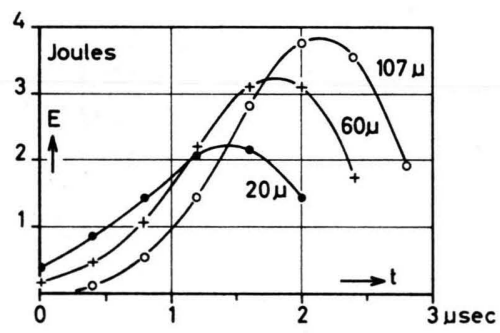

Fig. 7. Energie du plasma.

L'échelle de temps est relative.

Avant de calculer la température, considérons le rendement de chauffage. L'énergie maximum atteinte est de 3,75 Joules. D'autre part, l'énergie maximum emmagasinée dans les circuits électriques est donnée par $J^{2}{ }_{\text {eff }} / \omega^{2} C$, ce qui fait 2,0 Joules pour le système cubique et 2,25 Joules pour l'octaédrique. L'énergie totale délivrée au dispositif entier est donc de 8,0 Joules. Or le générateur programmé débite une puissance qui augmente presque linéairement avec le temps pour atteindre $13 \mathrm{MW}$ à la 10 e période. L'énergie produite par le générateur est ainsi de $\frac{1}{2} \cdot 13 \cdot 10^{6} \mathrm{~W} \cdot 2,1 \cdot 10^{-6} \mathrm{sec}=13,6$ Joules. Cela signifie qu'une fraction de $28 \%$ est transférée dans le plasma et qu'une fraction de $31 \%$ se transforme en énergie réactive. Le déficit est dû à une éventuelle désadaptation des circuits et au fait que d'autres dissipations d'énergie ont lieu dans le plasma, principalement l'ionisation.
La température moyenne est donnée par

$$
T=\frac{E}{3 k N} .
$$

Les températures électroniques et ioniques sont supposées égales. $N$ est le nombre d'électrons présents dans le plasma. Il est égal au produit du volume de la sphère et de la densité moyenne $n$. Cette dernière est déterminée par la pression de remplissage et le taux d'ionisation. Des mesures de densité n'ayant pas été faites, seul un ordre de grandeur peut être évalué. D'après des mesures faites par Quins ${ }^{10}$, on peut admettre que le taux d'ionisation est d'environ 50\%. La température moyenne calculée ainsi est reportée dans le Tableau 1. Dans ce tableau figurent également l'énergie maximum du plasma $E$, la presison moyenne $p$ et la pression maximum au centre $p_{\mathrm{c}}$.

\begin{tabular}{|c|c|c|c|c|r|}
\hline$p\left(\mathrm{H}_{2}\right)$ & $\begin{array}{c}E \\
\text { Joule }\end{array}$ & $\begin{array}{c}n \\
10^{21} \mathrm{~m}^{-3}\end{array}$ & $\begin{array}{c}T \\
10^{4} \mathrm{deg}\end{array}$ & $\begin{array}{c}p \\
n / \mathrm{m}^{2}\end{array}$ & $\begin{array}{c}p_{\mathrm{c}} \\
n / \mathrm{m}^{2}\end{array}$ \\
\hline $107 \mu$ & 3,75 & 3,7 & 4,7 & 4800 & 17200 \\
$60 \mu$ & 3,15 & 2,1 & 7,0 & 4000 & 13700 \\
$20 \mu$ & 2,2 & 0,7 & 14,5 & 2800 & 6200 \\
\hline
\end{tabular}

Tableau 1.

\section{Photographie du plasma}

La photographie ultra-rapide est effectuée avec une caméra électronique (convertisseur d'images) type EL 085/IPP, Garching bei München. La caméra est placée en direction d'un axe principal du système polyédrique.

Les Figures $8 \mathrm{a}$ à $8 \mathrm{c}$ * montrent trois séries d'instantanés pris à différentes pressions de remplissage et avec un temps d'ouverture de $0,2 \mu$ sec. Les temps indiqués sont relatifs au départ du générateur de haute fréquence. On détermine le temps zéro en photographiant une étincelle produite par le même générateur. La forte émission de lumière au centre provient de l'hydrogène, ce qui a été vérifié avec un filtre d'interférence $\mathrm{H}_{\beta}$.

D'autres photographies prises en régime de balayage sont assemblées dans les Figures $9 \mathrm{~b}$ à $9 \mathrm{~d}$. L'oscillogramme du signal du générateur (Fig. 9 a) est représenté dans sa position correcte par rapport à l'échelle de temps. On remarque le délai entre le maximum de la puissance $\mathrm{HF}$ et le maximum de la

\footnotetext{
* Figures 8 et 9 voir p. 1088 a.

10 J. M. P. Quins, Gas Laser Determination of the Electron Density in the Afterglow of a Hydrogen Discharge. Culham Reprint CLM-P 60, septembre 1964.
} 

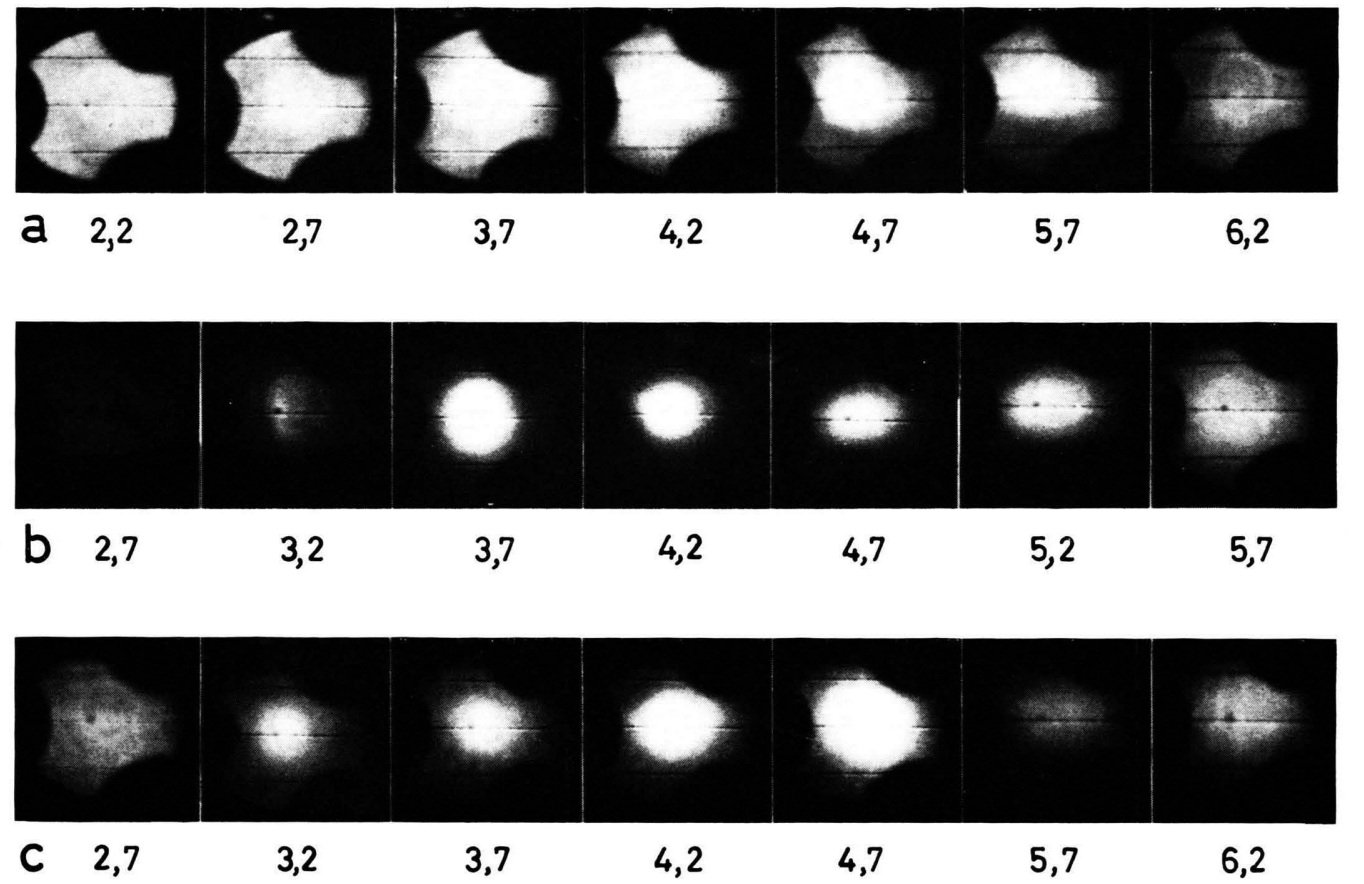

Fig. 8. Images successives de la région centrale. Les pressions de remplissage d'hydrogène sont dans l'ordre a, b et $\mathrm{c}: 107,60$ et $38 \mu$. Les instants d'ouverture de la caméra, indiqués en $\mu$ sec, sont relatifs au début du courant HF. La hauteur des photos correspond à $60 \mathrm{~mm}$.

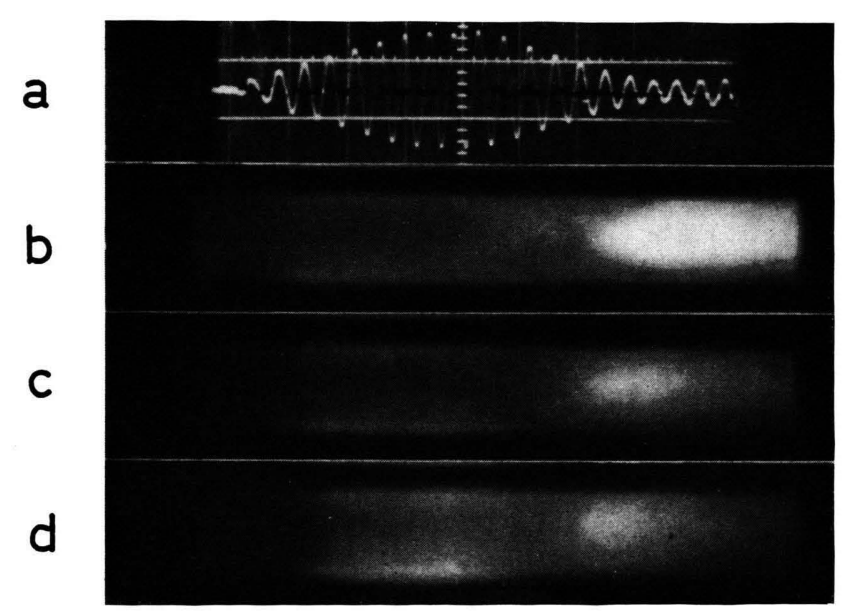

Fig. 9. Images balayées d'une fente placée devant le centre. a Oscillogramme de la tension au bobinage octaédrique. L'échelle de temps coïncide avec celle des photos. Fréquence: $4,7 \mathrm{MHz} ; \mathrm{b}$, c et d Pression de remplissage d'hydrogène 60,38 et $24 \mu$. Largeur de la fente $3 \mathrm{~mm}$. Longueur de la fente $34 \mathrm{~mm}$. 
brillance. Un ordre de grandeur de la vitesse de l'onde sphérique peut en être déduit si l'on divise le rayon du récipient par ce délai. A $60 \mu$ la vitesse ainsi obtenue est de $3 \mathrm{~cm} / \mu \mathrm{sec}$ tandis que la vitesse du son calculée pour la température moyenne correspondante, qui est de $7 \cdot 10^{4} \mathrm{deg}$, serait de $3,9 \mathrm{~cm} / \mu \mathrm{sec}$.
Ce travail a été effectué au Laboratoire de Recherches sur la Physique des Plasmas, financé par le Fonds National Suisse de la Recherche Scientifique. Nous devons notre reconnaissance à Monsieur $\mathrm{H}$. RIPPER pour la réalisation de la sonde de pression et pour son aide technique. 